\title{
A New Parallel Algorithm for Constructing Voronoi Tessellations from Distributed Input Data
}

\author{
D. P. Starinshak ${ }^{* a}$, J. M. Owen ${ }^{a}$, and J. N. Johnson ${ }^{b}$ \\ ${ }^{a}$ Lawrence Livermore National Laboratory, AX Division, M/S L-38, P.O. \\ Box 808, Livermore, CA 94550 \\ ${ }^{b}$ Lawrence Berkeley National Laboratory, Earth Sciences Division, 1 \\ Cyclotron Road M/S 74R316C, Berkeley, CA 94720
}

\begin{abstract}
We present a new parallel algorithm for generating consistent Voronoi diagrams from distributed input data for the purposes of simulation and visualization. The algorithm functions by building upon any serial Voronoi tessellation algorithm. The output of such a serial tessellator is used to determine the connectivity of the distributed domains without any assumptions about how points are distributed across those domains, and then in turn to build the portion of the global tessellation local to each domain using information from that domains neighbors. The result is a generalized methodology for adding distributed capabilities to serial tessellation packages. Results from several two-dimensional tests are presented, including strong and weak scaling of its current implementation.
\end{abstract}

\section{Introduction}

This article outlines a new algorithm for computing Voronoi and Voronoi-like tessellations in a robust and efficient manner from both shared and distributed input data. The design principle of the algorithm is unique in that any arbitrary serial method for computing Voronoi or Delaunay tessellations may be employed. The algorithm outlines a procedure for generating communication structures around serial tessellation packages to transform distributed input points into Voronoi tessellation data that is consistent across processor boundaries.

The introduction is subdivided into a discussion of Voronoi tessellations and terminology (1.1), a description of the computational framework for constructing Voronoi tessellations in shared and distributed-memory environments (1.2), and a summary of key application areas for distributed Voronoi construction (1.3). Section 2 outlines the parallel algorithm, including background information (2.1), a detailed statement of the algorithm (2.2), and remarks on efficiency improvements (2.3). Finally, Section 3 demonstrates results from a variety of two-dimensional unit tests, including strong and weak scaling of the new algorithm.

\footnotetext{
*Corresponding author. Email address: starinshak1@llnl.gov
} 

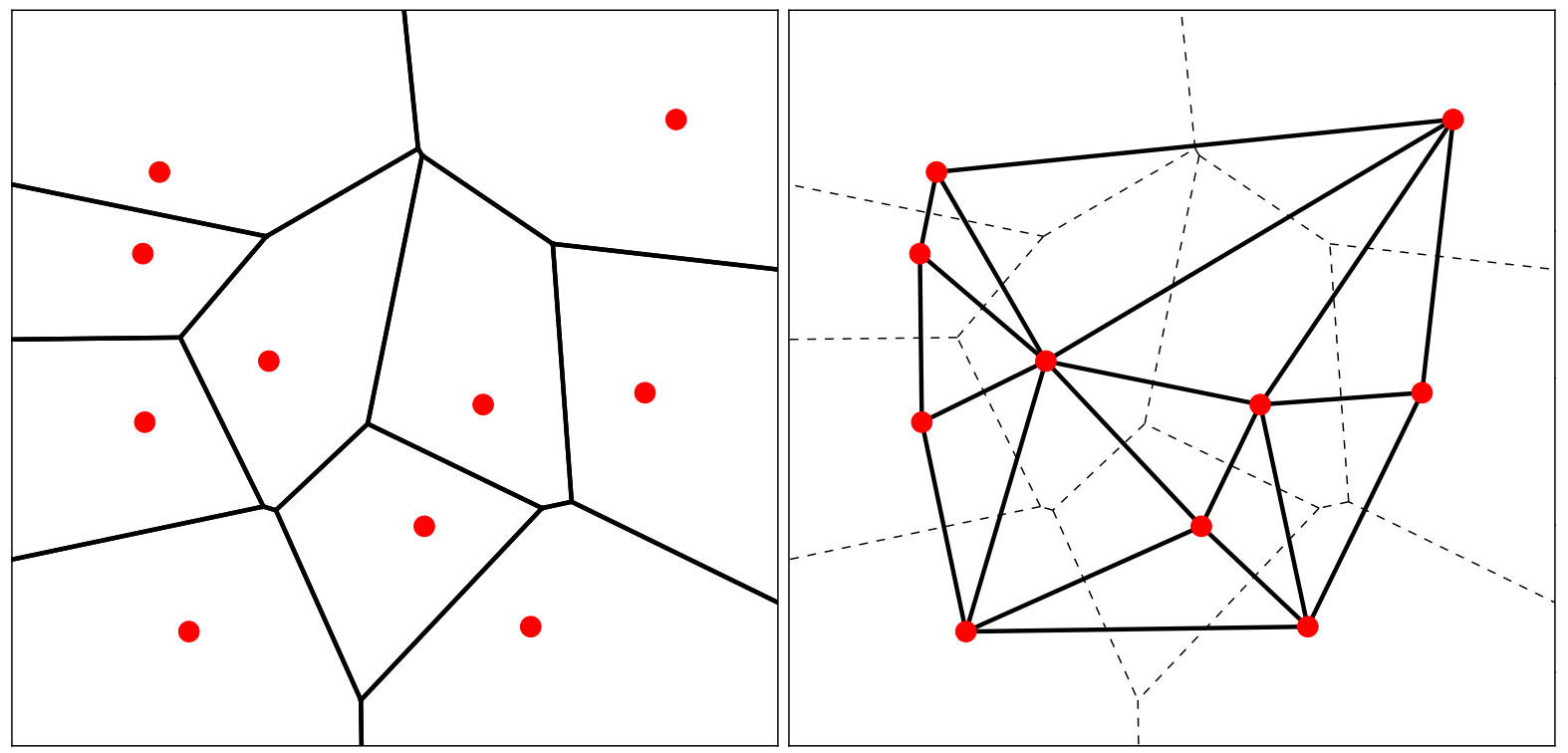

Figure 1: 2D Voronoi tessellation with generators (left) and related Delaunay dual tessellation.

\section{$1.1 \quad$ Voronoi Tessellation}

Let $\Omega \in \mathbb{R}^{N}$ define a closed region of space. A tessellation of $\Omega$ defines a set of disjoint cells whose union covers $\Omega$. Let $d(\cdot, \cdot)$ be a distance norm on $\mathbb{R}^{N}$, and let $\left\{\mathbf{g}_{i}\right\}_{i=1}^{G}$ be a collection of points inside $\Omega$. The Voronoi tessellation of $\Omega$ is given by the set of Voronoi cells $\left\{V_{i}\right\}_{i=1}^{G}$ with $i$-th cell corresponding to point $\mathbf{g}_{i}$ and satisfying

$$
V_{i}=\left\{\mathbf{x} \in \Omega \quad \mid d\left(\mathbf{x}, \mathbf{g}_{i}\right)<d\left(\mathbf{x}, \mathbf{g}_{j}\right) \quad \forall j \neq i\right\} .
$$

The points $\left\{\mathbf{g}_{i}\right\}_{i=1}^{G}$ are termed the generators of the Voronoi tessellation, and we denote their set $\mathcal{G}$.

The dual tessellation associated with the Voronoi is known as the Delaunay tessellation. Figure 11 illustrates an example Voronoi tessellation in two dimensions under Euclidean distance norm $d$ as well as its associated Delaunay dual. Voronoi cells are given by arbitrary polygons, and Delaunay cells are triangles. (In 3D, cells are respectively polyhedra and tetrahedra.) The mathematical relationship between Voronoi and Delaunay tessellations is a rich and interesting subject but outside the focus of this article. For our purposes, we simply observe that any Delaunay construction algorithm may be used to generate a Voronoi tessellation by computing the functional dual.

In this article, we limit discussion to two and three dimensions and consider only the Euclidean distance norm. Results and explanatory visuals are limited to two dimensions. The terms Voronoi tessellation, Voronoi diagram, and Voronoi grid are used interchangeably throughout this article. The details of Voronoi construction are based largely on geometric considerations. To that end, discussions often times refer to the geometric elements or data structures of a tessellation: namely, cells are composed of faces, edges, and nodes/vertices, and two neighboring cells in a tessellation share at least one of such elements.

Remark: Generalized Voronoi tessellations based on non-point generators may also be formulated, where distances in (1) are computed with respect to generator surfaces $\left\{\Gamma_{i}\right\}_{i=1}^{G}$ 
having dimension smaller than the global domain $\Omega$ [19]. While such generalized Voronoi tessellations are outside the scope of this work, the algorithm presented here should be applicable to parallelizing such generalized constructs as well.

\subsection{Problem Statement}

We seek an algorithm for constructing Voronoi diagrams based on distributed input generators. By "distributed" we mean that the global set of generators is subdivided and stored on separate processors. Each processor sees only its local subset of generators, and no generator is stored on more than one processor. We assume no memory is shared between processors. Furthermore, the parallel distribution of generators may be non-disjoint and no underlying geometric structure is assumed.

The geometric interpretation of (1) is that Voronoi cell $V_{i}$ consists of all points in $\Omega$ that are closer to its generator $\mathbf{g}_{i}$ than to any other generator. The difficulty of distributed-memory construction of this definition is apparent: the boundary of any local generator's Voronoi cell depends on the locations of all neighboring generators, whether their positions are stored on the local processor or not. Parallel communication between processors is therefore required to construct the consistent global Voronoi topology. Such communication should strive to be efficient, minimizing both the size and frequency of messages (i.e. generator positions) passed between processors.

The design principle of our parallel algorithm is to provide a generalized parallel interface to any serial or shared-memory construction algorithm. We establish parallel communication in order to determine a minimal but complete set of generator positions from neighboring domains such that each domain can construct the consistent subset of the global Voronoi diagram locally.

The number of serial construction algorithms for Voronoi and Delaunay diagrams are too numerous to mention here. (See [19] for an excellent summary.) Optimallyefficient implementations of many algorithms exist in the literature as do robust implementations in many popular two- and three-dimensional tessellation libraries (for instance, [23, 24, 26, 30]). We broadly divide such algorithms into two categories: direct and dual-based algorithms. Direct algorithms construct the Voronoi directly from input generators. Popular methods include incremental inclusion, divide-and-conquer, and plane-sweep methods such as Fortune's algorithm [8]. Dual-based algorithms compute the Delaunay diagram directly from input generators, then construct the Voronoi as its geometric dual.

At present, no direct algorithm for parallel Voronoi diagram construction has been formulated specifically for distributed-memory platforms. So-called "stitching algorithms" perform distributed construction by piecing together local tessellations computed on separate processors [11, 31]. However, such algorithms rely on a geometric decomposition of a stored global set of generators-a memory-intensive process for many scientific applications [17, 28, 18]. Numerous algorithms for direct Voronoi construction on shared-memory devices have been formulated, for instance [5].

By contrast, several parallel construction algorithms for Delaunay diagrams have been formulated for both shared-memory [2] and distributed-memory [3, 4, 15, 28] environments. Computing the Voronoi dual from a distributed Delaunay diagram is also discussed in [28]. For many of these algorithms, Delaunay construction is performed via divide-and-conquer into a series of subproblems; the algorithms compute the decomposition of a global point set for the goal of optimizing parallel scaling and load balancing. 
The resulting decompositions tend to be disjoint and convex.

The parallel algorithm we present is unique in that (i) it operates on any arbitrary spatial decomposition of the input generators, (ii) it does not rely on the global set of generators being stored on a single process, and (iii) it does not redistribute generators during parallel construction. The computed Voronoi diagram has a globally-consistent topology for arbitrary distributions and decompositions of input data.

Remark: Many serial Voronoi construction algorithms generalize to higher dimensions, non-Euclidean distance metrics, and non-point generators [19]. We note that the proposed parallel algorithm depends only on the resulting tessellations that such methods return, not on their underlying machinery. Thus our algorithm offers a means to potentially parallelize the construction of Voronoi tessellations having greater generality than those considered in this paper.

\subsection{Applications}

Applications for Voronoi tessellations as a mesh structure are numerous. As one of only a handful of automatic, on-the-fly methods for tessellating a domain, the Voronoi is a convenient mesh structure for mesh-free numerical methods or finite-volume-based methods having a dynamic mesh topology [17, 20, 21, 28]. Voronoi meshes are also widely used in Geographic Information Systems [13, 14] and subsurface flow calculations [18]. One particularly nice feature of the Voronoi for Lagrangian moving-mesh methods [17] is that, under continuous motion of the generating points, dynamic evolution of the Voronoi cells is smooth with respect to their volume topology. By way of comparison, dynamic Delaunay triangulation in general changes discontinuously (and globally so) as triangles flip edges.

Voronoi diagram generation is also robust to changes in length scale making it an attractive data structure for physical models that span several spatial scales, such as encountered in astrophysics, geophysical flows, and fluid dynamics [17, 20, 21, 28]. As an intuitive means of decomposing domains, Voronoi tessellations are a useful tool for visualizing discrete, spatially-distributed data and dynamically allocating resources in parallel molecular dynamics simulations [12, 22].

Applications for centroidal Voronoi diagrams are particularly numerous. The generators of such tessellations coincide with the geometric centroids of their associated Voronoi cells (see Figure 2). Centroidal tessellations satisfy a number of important optimality conditions, with applications ranging from image processing and data compression to data interpolation methods, point quadrature, and minimal-error finite differences. Consider [6] for an excellent overview of these ideas.

Uses for Voronoi diagrams as finite element meshes are explored in [7, 27]. Such meshes require high amounts of grid regularity to ensure numerical accuracy; the authors explore methods for efficiently optimizing Voronoi mesh geometry to that end. Similarly, the authors of [32] propose using boundary-restricted Voronoi diagrams to iteratively improve mesh isotropy. We note that iterative optimization schemes oftentimes require repeated construction of Voronoi diagrams to achieve convergence. Such schemes benefit greatly from parallel construction methods as a means to improve overall efficiency. 


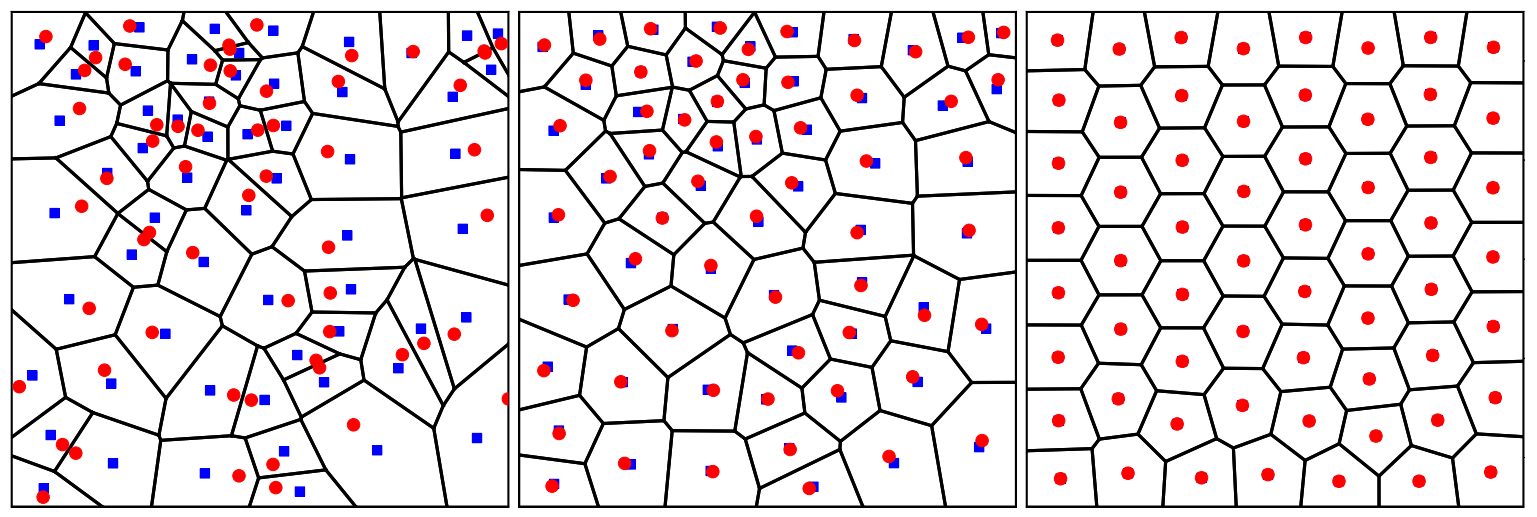

Figure 2: From left to right, a Voronoi tessellation after 0, 4, and 100 iterations of Lloyd's algorithm [16]. Generators (red circles) move towards cell centroids (blue squares) with each iteration. The tessellation slowly converges to a centroidal Voronoi diagram.
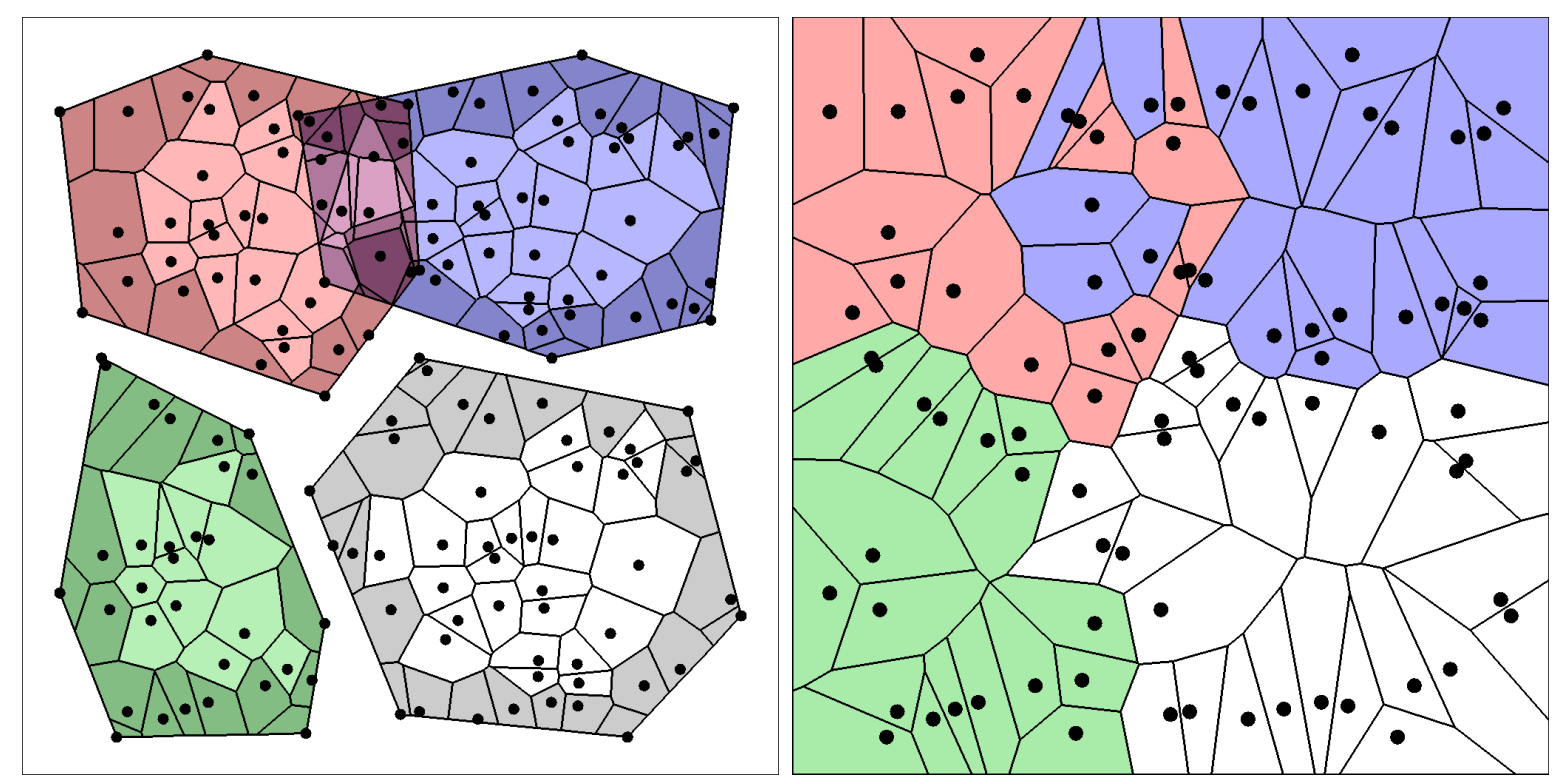

Figure 3: Diagrams generated to establish communication: local diagram on each processor, with visible generator cells shaded darker (left); and the visible mesh, identical on every processor, generated using the set of all visible generators to check adjacency (right). The top-right and bottom-left domains do not communicate.

\section{Parallel Algorithm}

This section describes the parallel algorithm for distributed Voronoi grid generation. The design principle of the algorithm is unique in that an arbitrary serial Voronoi or Delaunay construction method may be employed in its implementation.

\subsection{Background and Terminology}

We consider a distributed generator set: each processor sees only a local subset of the global generator set. The global set is never stored on any single processor. The parallel 

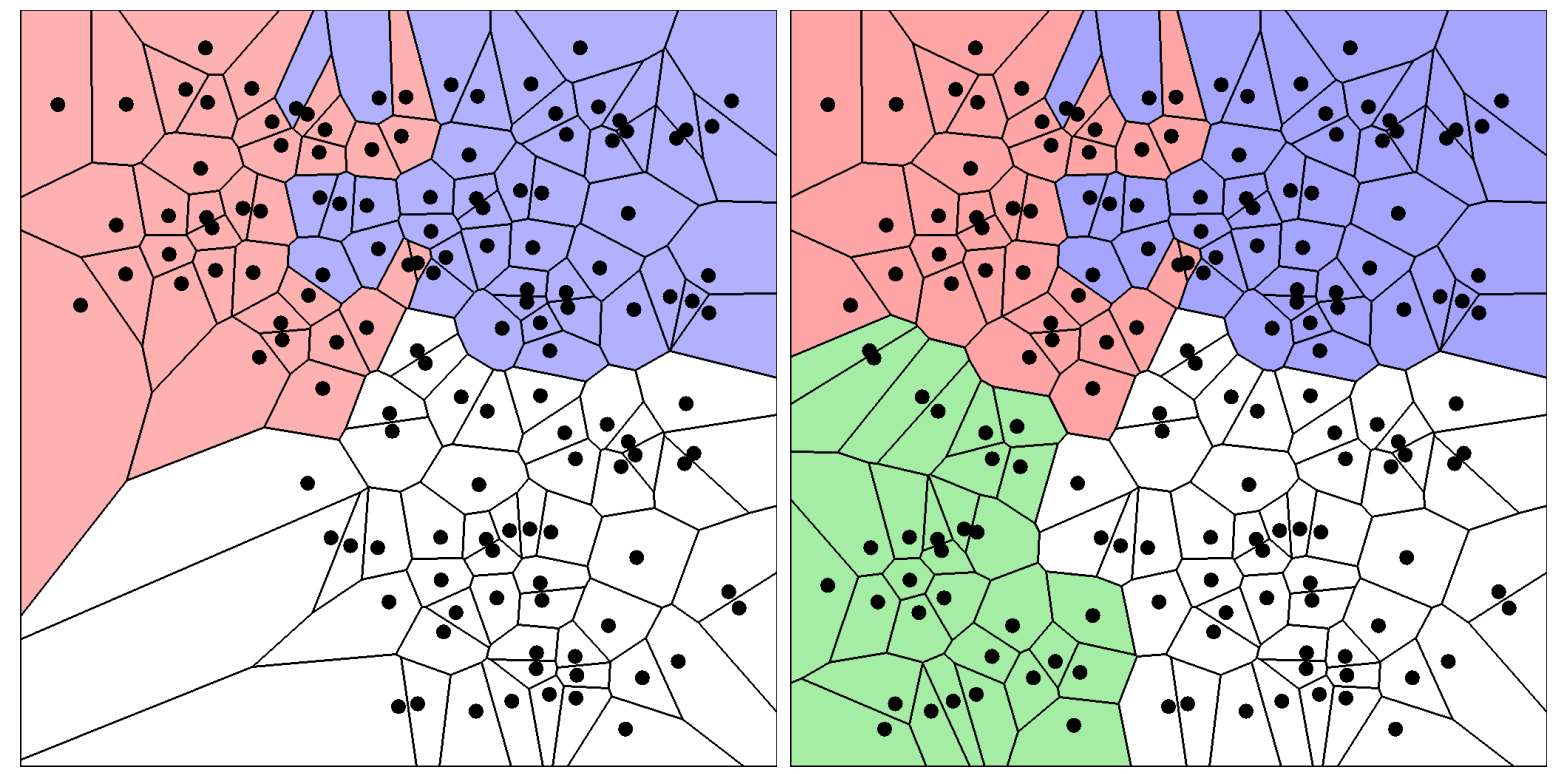

Figure 4: Diagrams generated post-communication: diagram constructed on the top-right processor using points from neighboring domains (left); cells from nonlocal generators will be deleted. The final diagram on each domain overlaid (right).

algorithm establishes communication between spatially-distributed (and not necessarily disjoint) domains of points. We say that two processors $P$ and $Q$ communicate or are domain neighbors if, in the global Voronoi diagram, there exists at least one generator stored on $P$ and one generator stored on $Q$ whose Voronoi cells share a Voronoi face, edge, or node. Using generator positions communicated from domain neighbors, a consistent subset of the full Voronoi diagram is computed locally.

We begin by defining terms. Let $\mathcal{G}$ be the global generator set and $\mathcal{G}_{P}$ the subset stored on processor $P$. Let $\mathcal{C}_{P}$ be the convex hull of point set $\mathcal{G}_{P}$. We define the local diagram on processor $P$ by the Voronoi diagram constructed from $\mathcal{G}_{P}$ and bounded inside its convex hull (see Figure 3, left).

To establish communication, we define the set of visible generators on each processor $P$, denoted $\mathcal{V}_{P}$. A generator is visible if its Voronoi cell in the local diagram has at least one node on the boundary. The visible sets for four different domains is illustrated in Figure 3 by shaded cells. Note that $\mathcal{V}_{P}$ always contains the vertices of convex hull $\mathcal{C}_{P}$; however, the set of hull vertices alone are not sufficient to establish communication. (See Section 2.3, Remark 1.) In general, for $\mathcal{O}(N)$ local generators in $2 \mathrm{D}$, the visible set is of size approximately $\mathcal{O}\left(N^{1 / 2}\right)$ points.

The visible set is used to determine communication. In particular, if two domains are disjoint (i.e. their local convex hulls are non-intersecting) but neighbor one another, adjacent cells on separate domains correspond to visible generators.

The argument for this is straightforward. Suppose processors $P$ and $Q$ communicate such that the Voronoi cell of generator $\mathbf{g}_{P}$ on $P$ neighbors the Voronoi cell of $\mathbf{g}_{Q}$ on $Q$ in the global diagram. Further, assume $\mathcal{C}_{P}$ and $\mathcal{C}_{Q}$ are disjoint. (We return to the non-disjoint case later.) Finally, suppose neither $\mathbf{g}_{P}$ nor $\mathbf{g}_{Q}$ are visible. This implies that all of their local Voronoi nodes lie completely inside their respective processors' convex hulls.

Let $\mathbf{x}$ be a point on the shared boundary between Voronoi cells of $\mathbf{g}_{P}$ and $\mathbf{g}_{Q}$. We 
show that the existence of $\mathbf{x}$ contradicts the two generators not being visible:

- $\mathbf{x}$ cannot be inside the local Voronoi cell of $\mathbf{g}_{P}$ or on its boundary. Otherwise, $\mathbf{g}_{Q}$ would also be inside $\mathcal{C}_{P}$, contradicting the fact that the convex hulls are disjoint.

- $\mathbf{x}$ cannot be outside the local Voronoi cell of $\mathbf{g}_{P}$. This would make $\mathbf{x}$ closer to some other generator in $\mathcal{G}_{P}$ than to $\mathbf{g}_{P}$, thus violating the Voronoi property (1) locally.

The same reasoning applies to $\mathbf{g}_{Q}$. If $\mathbf{x}$ can be neither inside nor outside either of two generators' local cells, then $\mathbf{g}_{P}$ and $\mathbf{g}_{Q}$ cannot share a Voronoi node in the global diagram. We arrive at a contradiction unless at least one of $\mathbf{g}_{P}$ and $\mathbf{g}_{Q}$ is visible.

\section{$2.2 \quad$ Algorithm Statement}

Each processor first computes its local Voronoi diagram and determines its set of visible generators. This can be accomplished by finding all local Voronoi cells intersected by the planar segments composing the local convex hull boundary.

Once computed, each processor then sends its visible set to every other processor. Although the potential exists for this to be a large number of point, we note that it is a dimensional reduction from the full local set: only points on the bounding surface of the processor are shared, not those on its interior volume. For large numbers of processors, this step may lead to a computational bottleneck for the algorithm. (See Section 2.3, Remark 4.)

At this point, processor $P$ has access to the visible set from all other processors as well as their convex hulls, by the definition of $\mathcal{V}$. Each processor then computes a second local Voronoi diagram using the full set of shared visible generators. We refer to this as the visible diagram and note that it is guaranteed to be identical on every domain (see Figure 3 , right). $P$ establishes communication by performing two tests locally:

(i) Hull Intersection: If convex hulls $\mathcal{C}_{P}$ and $\mathcal{C}_{Q}$ intersect, then $P$ and $Q$ likely communicate.

(ii) Adjacency: If a cell whose generator belongs to $P$ is adjacent to a cell whose generator belongs to $Q$ on the visible diagram, then $P$ and $Q$ communicate.

Any processor $Q$ satisfying either (i) or (ii) are added to $P$ 's list of neighboring processors. It is worth emphasizing these definitions are chosen for robustness and correctness - how efficient these criteria are at restricting the domains that interact as neighbors is a function of how the generators are domain decomposed. For instance, if generators are randomly assigned to processors likely these criteria will indicate all processors are in communication. To improve the efficiency of such cases one should more reasonably domain decompose the generators: however, regardless of how the generators are distributed we expect the algorithm to always return the correct tessellation.

Each processor now maintains a consistent list of its neighbors. A second and final communication stage is performed: each processor shares its full generator set with each of its neighbors. A final Voronoi diagram is then constructed using the set of local generators plus the generators from each of the neighbors (see Figure 4 , left). Voronoi faces, edges, and nodes are accumulated, and the neighbor list is revised (if necessary) based on the existence of shared elements. Cells not belonging to the local domain are deleted, leaving behind a local Voronoi diagram whose topology is consistent across processor 
boundaries (see Figure 4, right). We summarize the algorithm below.

\section{Parallel Algorithm:}

\section{Stage 1: Establish Communication}

1. Construct local Voronoi diagram using local generator set, bounded by convex hull.

2. Compute set of visible generators and share with all other processors. Note this stage includes communicating the convex hulls.

3. Construct visible Voronoi diagram using the set of all visible generators. (Diagram is identical on each processor.)

4. Evaluate tests to establish domain neighbor list:

(i) Hull intersection: does another domain's convex hull intersect the local hull?

(ii) Cell adjacency: does a Voronoi cell from another domain neighbor any local cell in the visible diagram?

\section{Stage 2: Compute Final Diagram}

1. Send local generator set to neighbors.

2. Construct Voronoi diagram from local generators plus neighboring domain generators.

3. Identify shared faces, edges, and nodes and revise (reduce) the neighbor list if necessary.

4. Delete nonlocal cells from diagram.

Remark: Note that hull intersection does not imply communication in general. It is possible for the test to return a "false positive" for non-convex decompositions of the global generator set. (For instance, consider concentric rings of generators, with each ring assigned to a separate domain.) For the sake of robustness in the algorithm, we allow positive hull intersection to imply communication, at the potential cost of more communication than strictly necessary. This is only an issue for pathological domain decompositions.

\subsection{Remarks on Efficiency}

\section{Remark 1: Sharing Convex Hull Vertices Instead of Visible Points}

The set of visible points on a processor may be large, especially when generators are clustered close to the convex hull. This can severely impact parallel efficiency for large numbers of processors. It is reasonable to ask whether we really need to share all visible points. Can we get by sending only the convex hull vertices? Unfortunately the answer is no, as illustrated in Figure 5. The bottom of three disjoint domains has a visible point near the boundary of its convex hull (left). The top and bottom domains share a common edge on the global diagram (right). However, if only convex hull points are shared when computing the visible diagram (center), the middle domain can "shield" the 
top and bottom domains from seeing one another in the adjacency test. In general, an arbitrary number of cells on the visible diagram may shield two neighboring processors from seeing one another.
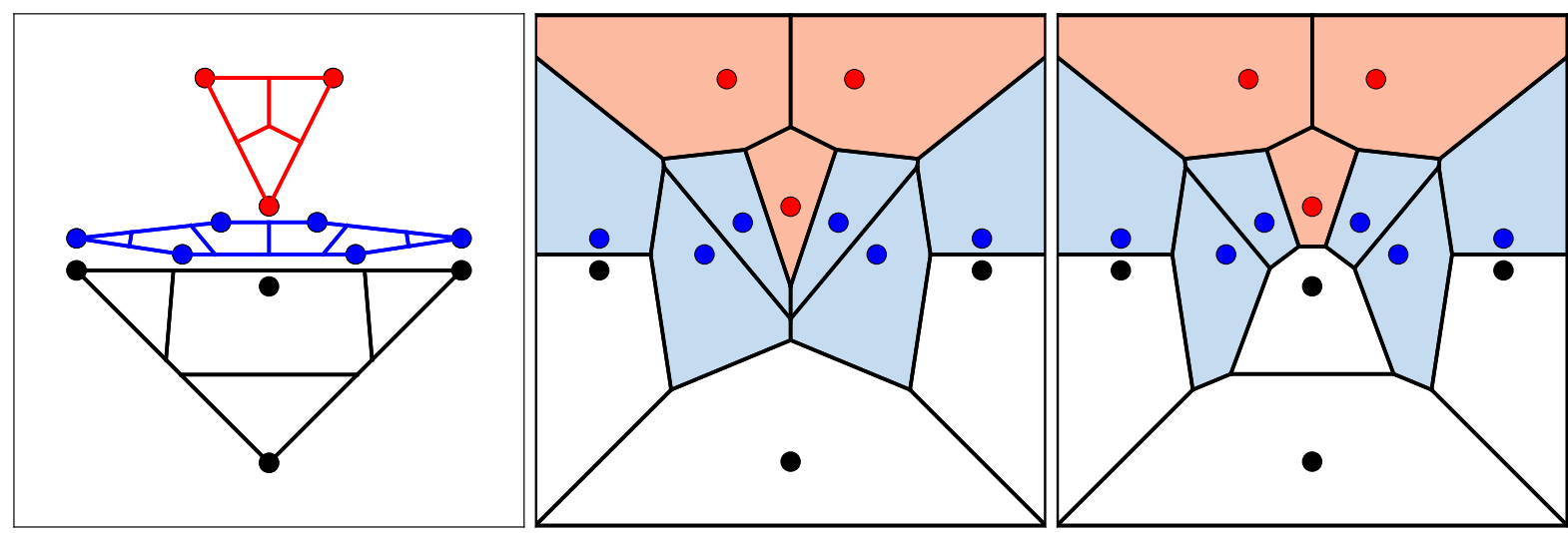

Figure 5: Local diagrams on each convex hull, colored by processor (left) and the final correct diagram (right); the diagram composed only of convex hull vertices, and excluding the visible set, (center) does not capture communication between the top and bottom processors.

Remark 2: Communicating Only the Visible Points Sharing the full set of local generators with all neighboring processors is sufficient to construct a consistent Voronoi topology, but it is not necessary. Using a smaller subset would decease message size and improve parallel efficiency, especially for large numbers of processors. It is reasonable to ask whether the visible set itself constitutes a sufficient subset of points. Unfortunately, the answer is again no.

It is easy to see in the case of neighboring processors having intersecting convex hulls: non-visible generators on a processor can clearly neighbor Voronoi cells on another processor in the global diagram. The case of disjoint neighboring processors is less obvious. Consider Figure 6, a point can be on the interior of its processor's local convex hull (i.e. not visible) but be close enough to a point on a neighboring processor (left) to influence its Voronoi cell on the global diagram. If the bottom processor shares only its visible set (center), then the neighboring processor will compute an incorrect Voronoi topology locally. Sharing the full set (right) results in a globally-consistent topology.

It may be possible for each processor to compute a sufficient subset of generators to share with neighbors by computing a second visible diagram using the full set of local generators and the visible sets of all its confirmed neighbors. This hypothesis is not yet tested; we regard it as part of future efficiency improvements for the parallel algorithm.

\section{Remark 3: Improving the Hull Intersection Test}

The hull intersection test may be narrowed. For processors $P$ and $Q$, a better test would check if any point in the visibility set of $P$ is inside $\mathcal{C}_{Q}$ and vice versa. This potentially reduces the number of "false positives" in the communication list and cuts back on the total number of send/receive operations.

Remark 4: Removing the All-to-All Communication 

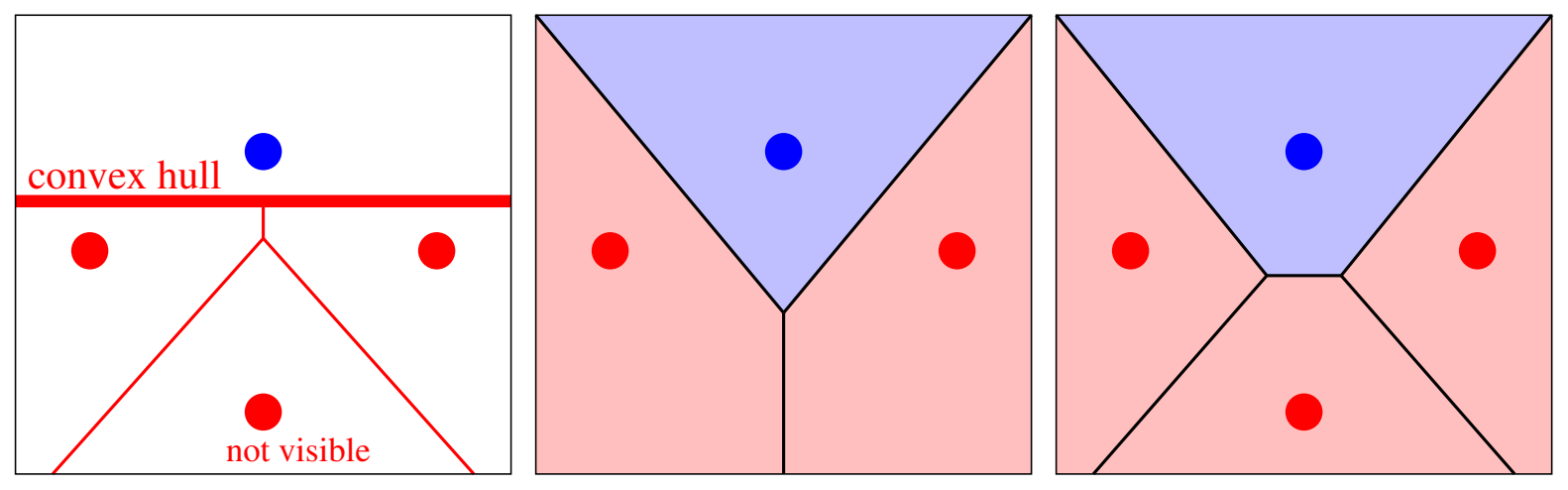

Figure 6: Zoom in of the convex hull boundary of local Voronoi tessellation and a single generator from a neighboring processor (left). The Voronoi diagram computed on the neighboring processor is incorrect if the first processor only shares its visible set (center) compared to sharing its full set (right).

Sharing the set of visible points with every processor is not mandatory for the algorithm to succeed. Preprocessing may be done to approximate a domain's communication list, for instance by quantizing processor locations using a global octree. Such a strategy would improve efficiency for massively-parallel computations and speed up the construction of the visible diagram on each processor.

\section{Remark 5: Dynamic Generators}

An important application of this algorithm is repeated and on-the-fly Voronoi mesh generation for distributed, dynamically-evolving generator positions. This can routinely occur in Lagrangian-frame hydrodynamic simulations [17, 20, 21, 28].

As generators move, domain neighborhoods change. The work needed to establish communication between processors can increase as more domains have intersecting convex hulls or the number of visible generators on a processor increases. (See the Taylor-Green Vortex example in Section 3 for an extreme example of this.) Although the parallel algorithm is less efficient when this occurs, the actual inefficiency is a function of how the algorithm is applied not of the algorithm itself. The algorithm is designed to be agnostic to the distribution of generators across domains; it will compute a valid mesh topology regardless of the point decomposition.

We consider two simple efficiency improvements in the context of moving generators:

Dynamic Partitioning of Generators

One can migrate generators across processors. For instance, one may construct a static spatial partition in the background that all processors know about; if a local generator moves outside a partition boundary to a new partition region, the generator is sent to the domain owner of that partition region. This type of dynamic load balancing is routinely used in parallel particle-based hydrodynamic simulations [12, 20, 22].

It is important to point out that a static background partition does not solve the problem of constructing a parallel-consistent Voronoi. Two processors can have neighboring Voronoi cells on the global diagram even if they don't have neighboring regions in the static partition. This is especially true if there is a large range in generator density/clustering. 
Reusing Domain Neighborhoods

If generator positions do not move appreciably during an update cycle, the domain neighborhoods should not change appreciably during that cycle either. In this case, Stage 1 of the parallel algorithm need not be repeated. Each domain could conceivably use the neighborhood computed in the previous cycle (or even overestimate the new neighborhood by including the "neighbors of neighbors" from the previous cycle) and skip directly to Stage 2 or the algorithm.

Note that this is a risky procedure in practice. There is no a priori metric for whether a generator has moved an "appreciable" distance that its domain's neighborhood has definitively changed.

\section{Results}

The following set of results were obtained using Polytope, an open-source library for Voronoi grid generation [34]. An implementation of the parallel algorithm exists within Polytope.

In addition, many of the generated tessellations conform to a given input boundary (from simple boxes to complicated, non-convex figures). In each case, an unbounded Voronoi diagram is computed using generators lying inside the input boundary. Voronoi cells are then clipped by the boundary geometry, in the spirit of [32, 33], returning a boundary-conformal tessellation. Note that in the case of non-convex boundaries, the tessellation may no longer be strictly Voronoi along those boundaries.

\section{Example Boundaries}

Figure 7 presents a collection of complicated 2D example boundaries. In most cases, 2000 random generator positions are distributed across 36 processors. Color changes indicate domain boundaries. The parallel algorithm respects non-convex regions, and Voronoi topology is consistent across domain boundaries.

\section{Centroidal Relaxation}

Centroidal Voronoi diagrams possess a number of attractive properties for applications in image processing, discrete statistics, and finite element quadrature [6]. Further, dynamic simulations computed on centroidal Voronoi meshes benefit from approximately-equal angles and aspect ratios within cells [17].

Parallel construction significantly improves the efficiency of iterative optimization schemes which attempt to relax generator positions to cell-centroid locations. Capabilities are demonstrated on a toroidal boundary, with 4000 generators distributed over 20 processors. The diagram is relaxed using 1000 iterations of Lloyd's algorithm [16]. Figure 8 gives the log of the condition number for each cell before and after relaxation as a relative measure of grid optimization [9]. Cell shapes approach regular hexagons, and the condition number approaches a constant value everywhere. 


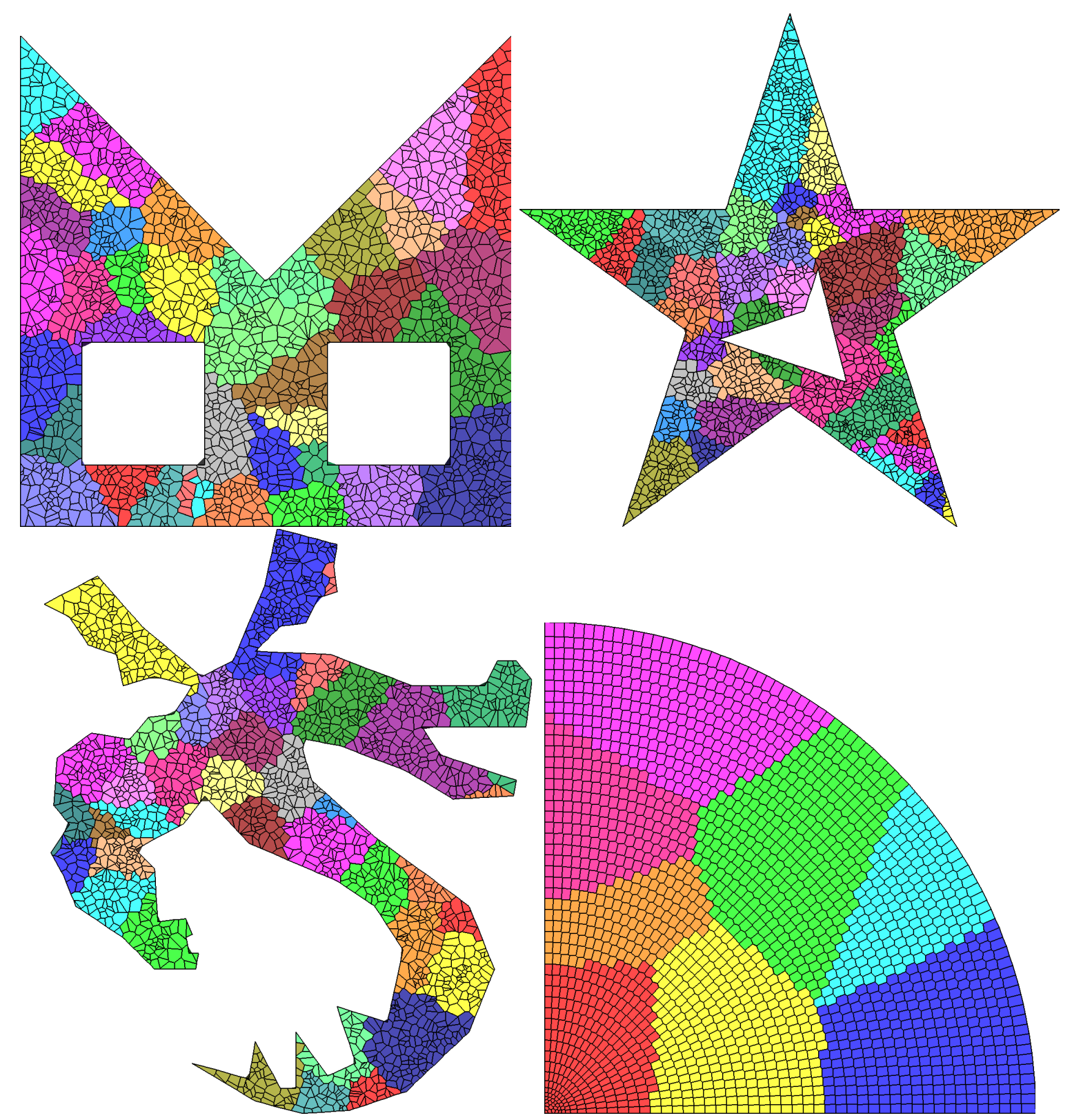

Figure 7: Distributed diagram computed on a collection of complicated boundaries. Colors indicate different computational domains.

\section{Moving Generators: Taylor-Green Vortex}

The following test demonstrates the robustness of the parallel algorithm. $50 \times 50$ generators initially on a unit lattice are decomposed disjointly onto 16 domains. The generators are then moved smoothly following a prescribed background velocity from the TaylorGreen vortex [29]. This test highlights the robustness of the parallel communication algorithm since each update of the generator positions can lead to changes in processor communication. Voronoi meshes at times $0,0.5,2$, and 4 are given in Figure 9 .

Note that while the original domain decomposition at time 0 (upper-left panel of Figure 9p allows our algorithm to successfully reduce the number of processors communicating with one and other, by the end state (in the lower-right panel) essentially all 


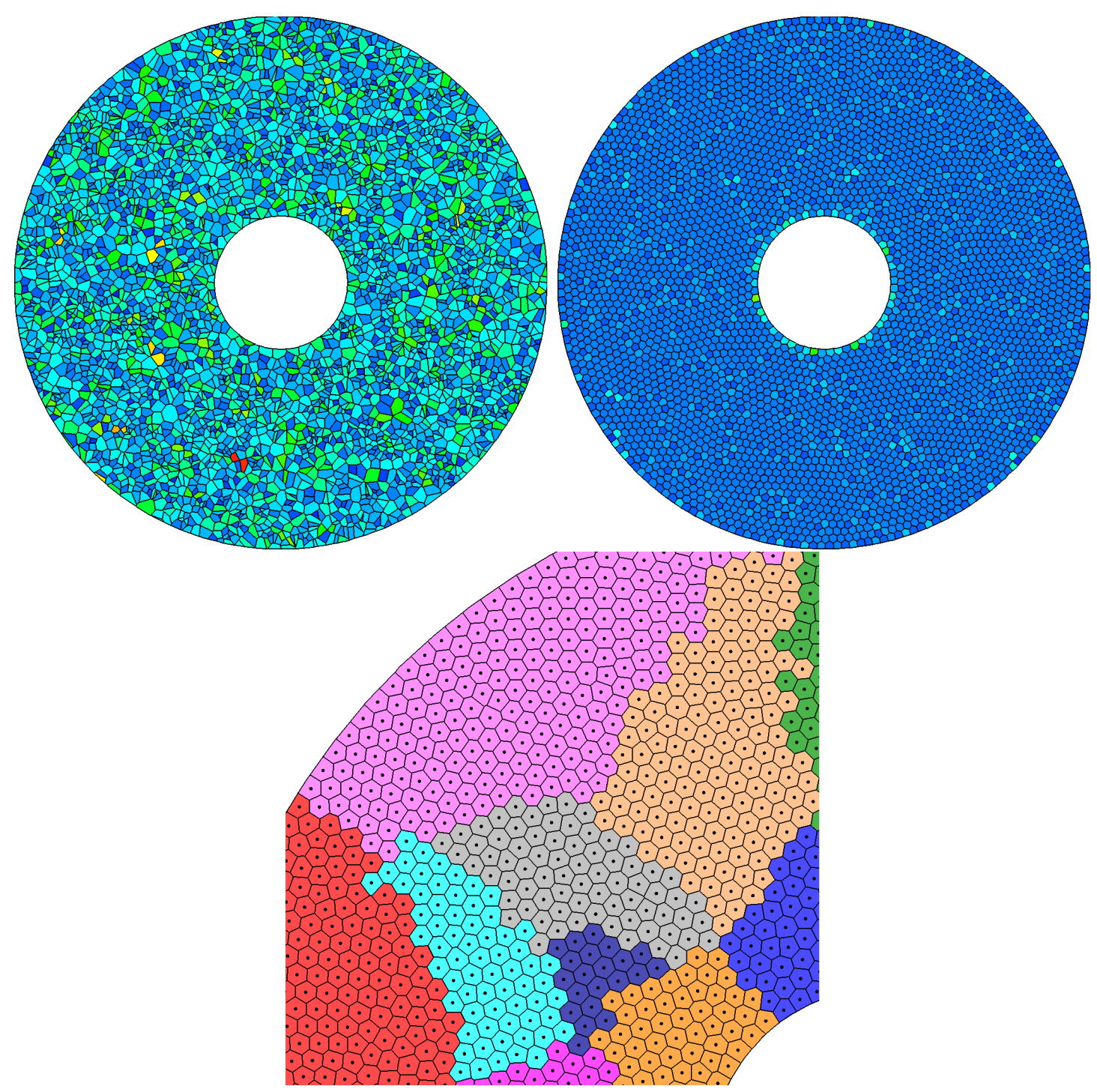

Figure 8: Centroidal relaxation performed on 4000 input generators distributed over 20 processors. Log condition number plotted for the initial mesh and after 1000 iterations of Lloyd's algorithm (top). Zoom in of the relaxed mesh showing domain decomposition (bottom).

domains are in communication. While we lose the efficiency of parallel Voronoi generation as the problem proceeds, it is a nice demonstration of how the algorithm remains robust as we transition from a reasonable domain decomposition to a pathological one.

\section{Scaling Study}

We present parallel scaling results for the problem of meshing a unit square using $G$ input generators on $N$ processors. Three domain decompositions are considered and illustrated in Figure 10.

Optimal: generators and domains are laid out on a Cartesian lattice, with equal numbers of points per processor.

Unbalanced: points are randomly-distributed and assigned to processors to give 

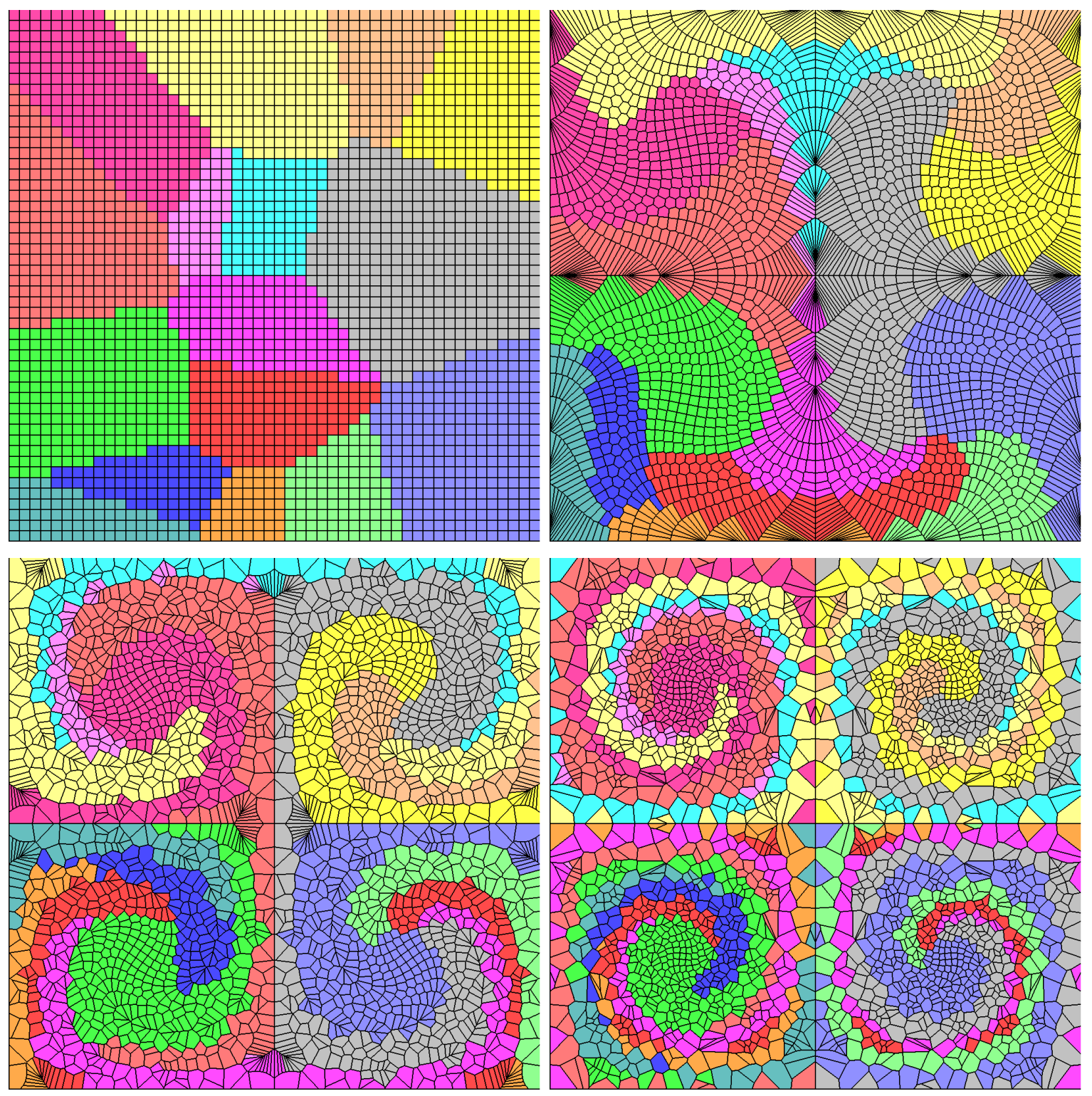

Figure 9: $50 \times 50$ generators distributed over 16 domains. Generator positions advect according to a Taylor-Green vortex velocity field. Voronoi meshes at times 0, 0.5, 2, and 4. Colors indicate domain index.

spatially-disjoint domains (as in Figure 7). Work is not balanced between domains; generators per processor can differ by more than a factor of two.

Random: points are randomly-distributed and assigned randomly to processors. This is the worst-case scenario for our algorithm, as all processors must communicate with one another to form a valid topology.

Results from two different serial Voronoi tessellators are presented: a dual method using the Delaunay tessellator Triangle [24] and a direct method using the Boost.Polygon Voronoi library utilizing Fortune's optimal sweep algorithm [8, 30]. As expected, the serial overhead is much smaller for the latter algorithm. 

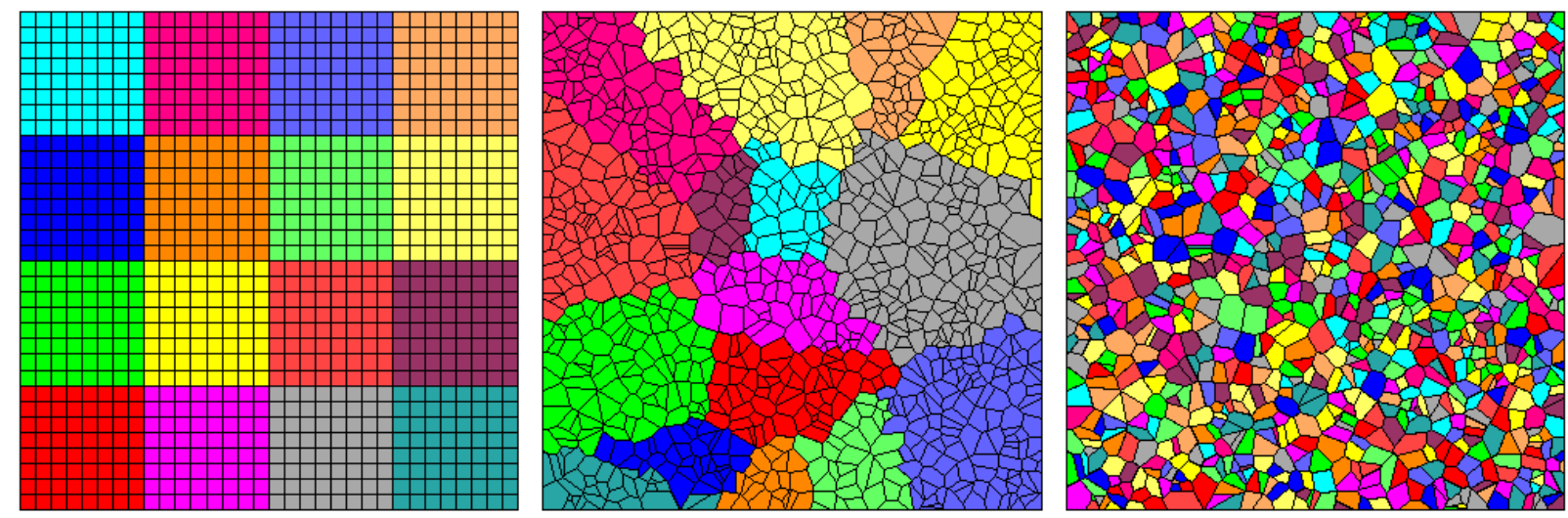

Figure 10: Decompositions of 1024 points onto 16 processors to test different scaling regimes: optimal (left), unbalanced (center), and random (right).

\section{Strong Scaling:}

Strong scaling results are presented in Figure 11. $G=640,000$ generators are assigned to $N$ processors such that per-processor workload decreases and communication increases in $N$. For the unbalanced decomposition, the number of generators per processor is not equal.

The various decompositions perform as expected. The random case leads to no parallel speedup: all domains communicate, causing processors to compute identical meshes as if in serial. For the other decompositions, total time decreases as per-processor workload decreases, with the optimal case outperforming the unbalanced case. The trend flattens out for larger numbers of processors indicating a larger and larger communication cost. Exploring this trend for processes numbering greater than 256 is a source of future work.

\section{Weak Scaling:}

Weak scaling results are presented in Figure 12. $G$ scales with the number of processors such that each domain is assigned 2500 generators (exactly 2500 for random and optimal decompositions and 2500 on average for the unbalanced case). The scaling trend, normalized by generators per processor, was found to converge for generators numbering greater than 2500 .

The random decomposition again exhibits serial scaling, as total calculation time is linear in the number of processors. The optimal case outperforms the unbalanced case, as expected. However, both trends are monotonically increasing in process count, indicating calculation costs are dominated by communication.

We would like to point out that the weak scaling results, while certainly not optimal, are not necessarily algorithmic in nature. Current implementation of the parallel algorithm has focused on robustness and generality. Work has not been done to profile the parallel algorithm or optimize its implementation at the time data was taken. Overall, the algorithm demonstrates the ability to compute consistent Voronoi grid topologies on large numbers of processors without failure. Optimization of its implementation is ongoing work; initial ideas for improving parallel scaling are provided in the closing remarks of Section 2 . 


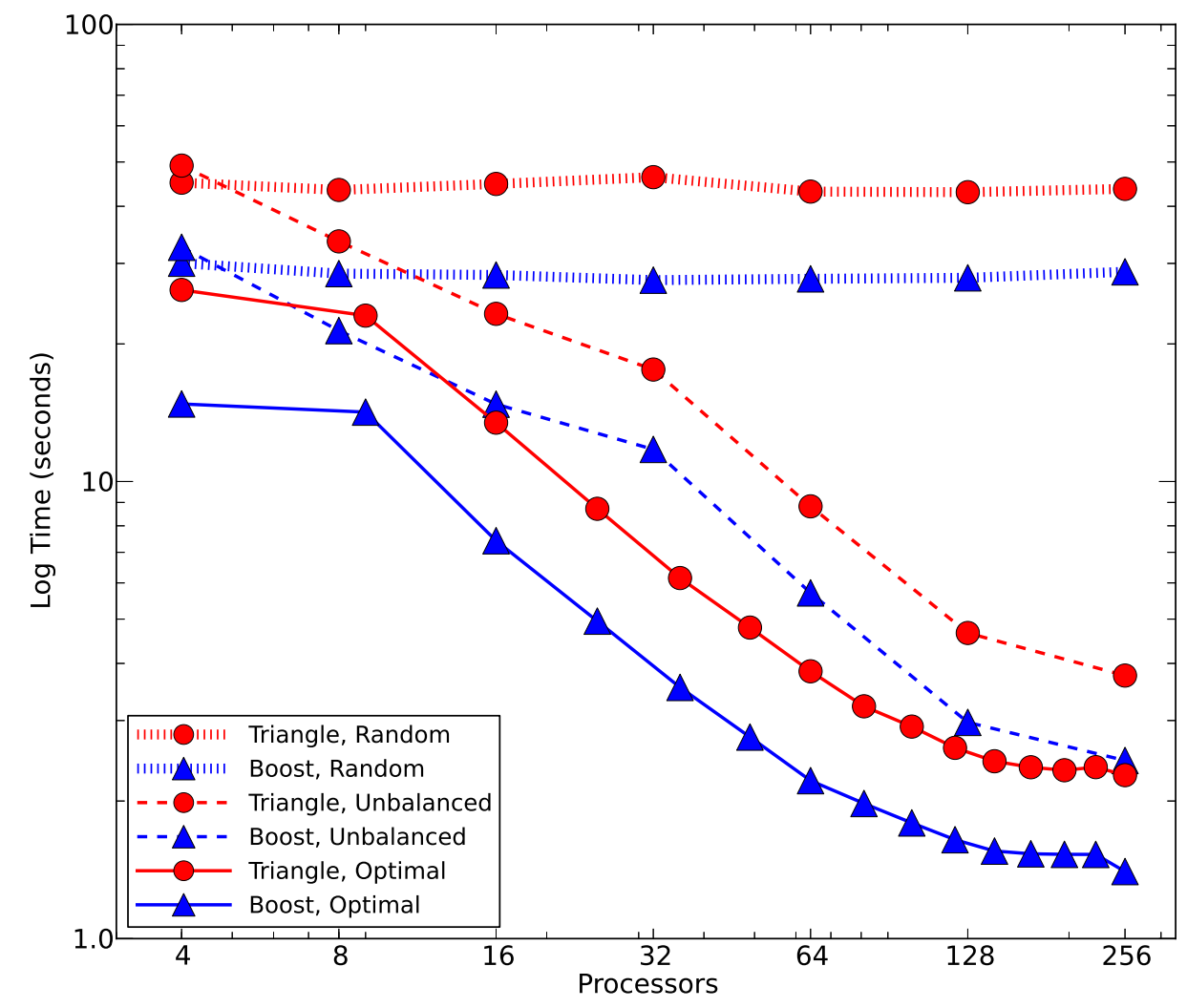

Figure 11: Parallel strong scaling of 640,000 generators on $N$ processors. Generators uniformly distributed in a unit box. Comparison of reasonable and random domain decompositions.

\section{Timing Profile:}

We repeat the strong scaling test with $G=10^{6}$ generators on a lattice having optimal decomposition. The individual steps of the parallel algorithm described in Section 2.2 are timed and presented in Figure 13. Results are organized based on the two algorithm stages: establishing the set of neighboring processors (left) and constructing the final distributed Voronoi diagram using communicated generators from neighbors (right). The five most expensive steps of the algorithm are presented in Figure 14 based on percentage of total mesh construction time. The Boost.Voronoi serial tessellation library is used for this test. A number of conclusions may be drawn from the data.

First, the strong scaling results for Stage 2 of the algorithm are excellent. If the processor neighbor set is known a priori and Stage 1 need not be exercised (for instance, by reusing a previously computed set, as in the case of dynamic generators) then the right panel of Figure 13 gives the appropriate strong scaling for the algorithm.

Second, the three most expensive operations all involve computing serial tessellations:

- First is constructing the local diagram to find visible generators (Figure 13, left panel, blue curve). This operation scales well here: more processors implies fewer local points. 


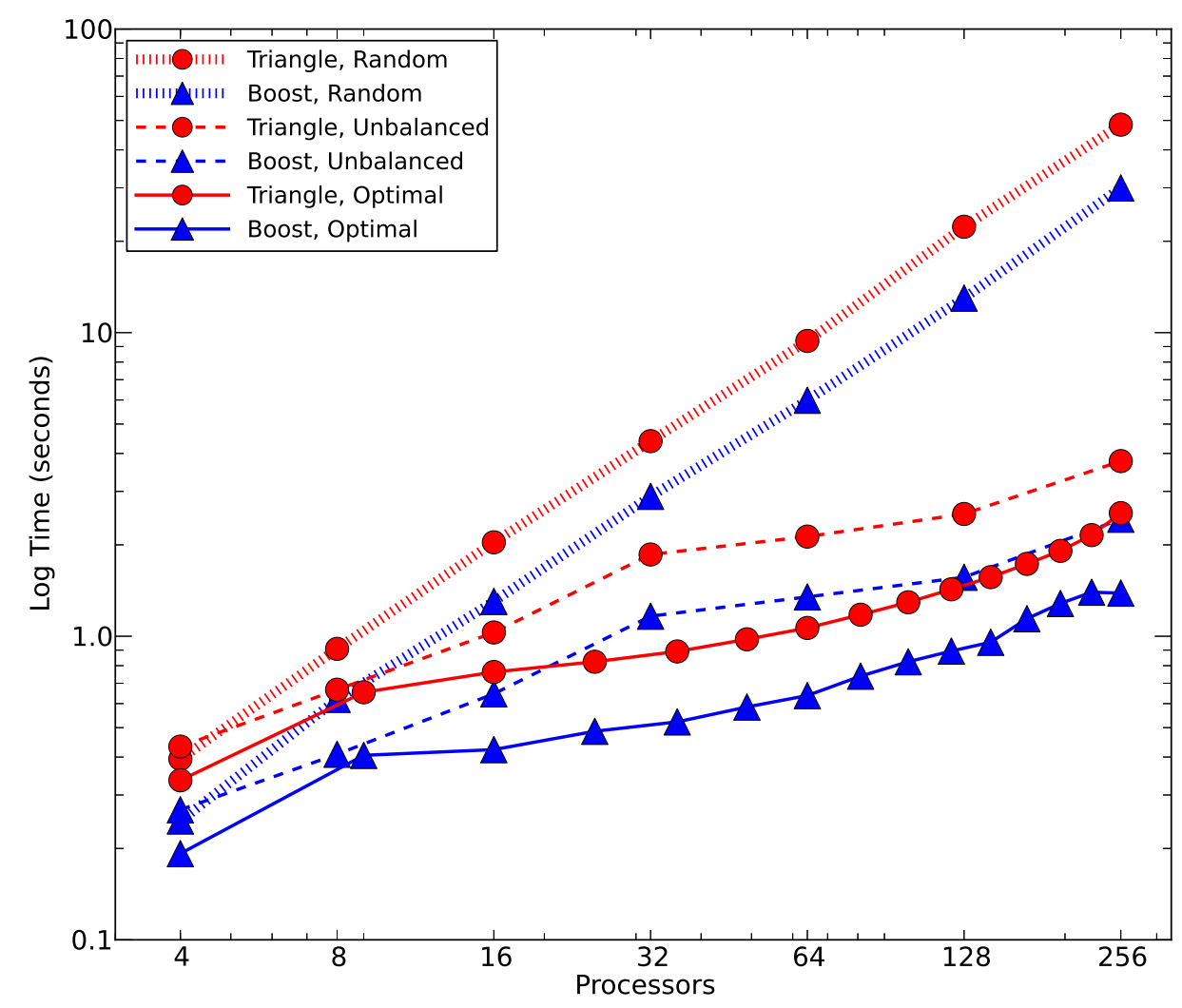

Figure 12: Parallel weak scaling for approximately 2000 generators per processor. Generators uniformly distributed in a unit box and decomposed into domains so that domain hulls are non-overlapping.

- Second is constructing the visible diagram from the visible generators on every processor (Figure 13, left panel, red curve). This operation does not scale well due to the all-to-all communication of the visible set: more processors implies a much larger visible diagram.

- Third is constructing the Voronoi diagram using all local generators and all generators communicated from neighbors (Figure 13 , right panel, green curve). This is the most expensive operation for small decompositions since every processor likely communicates with every other processor but ultimately scales well.

Finally, results indicate that parallel scaling would indeed benefit from the efficiency improvement ideas presented in Section 2.3, namely Remarks 3 and 4 . Cutting down on the all-to-all communication using a global octree would reduce the number of generators involved in computing the visible tessellation and improve its timing as decomposition size increases. Likewise, improving the implementation of the hull intersection test, while currently not a significant percentage of total Voronoi construction time, would nevertheless improve its scaling. These modifications are a source of future work. 

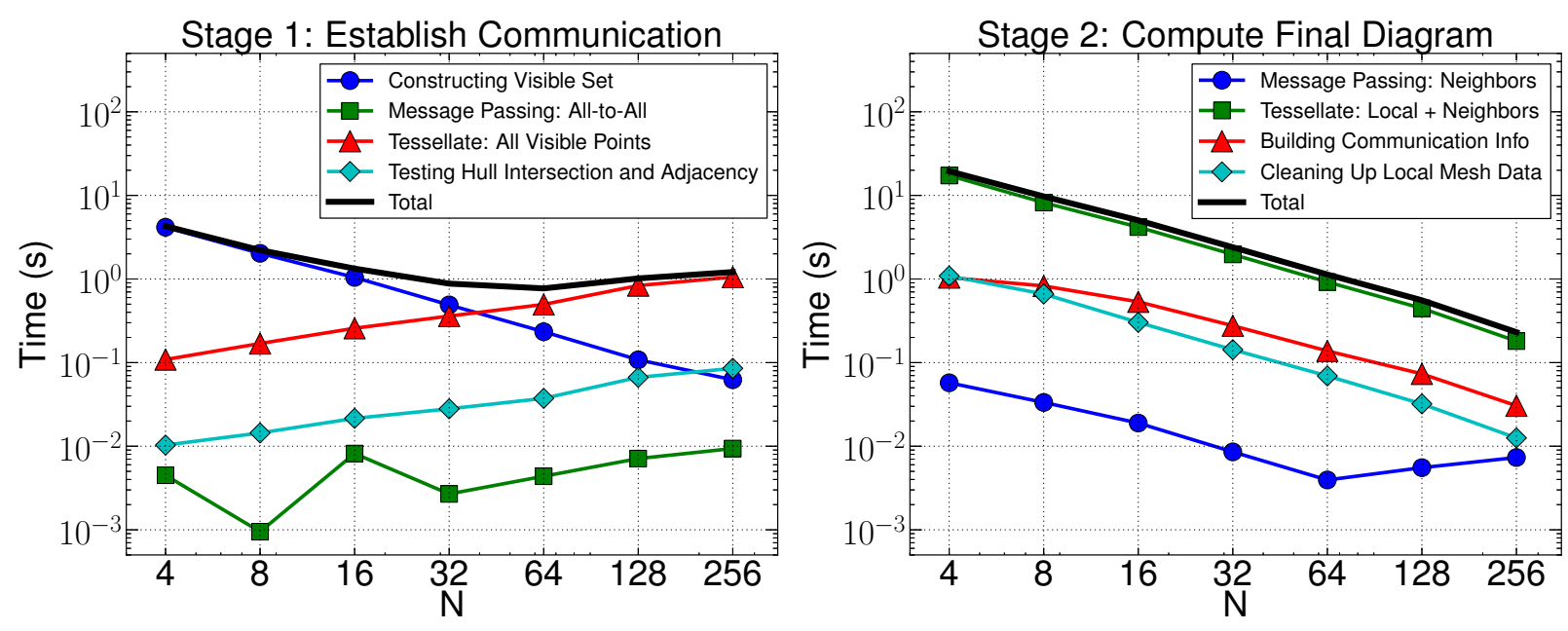

Figure 13: Parallel strong scaling of $10^{6}$ generators on a lattice distributed across $N$ processors with optimal decomposition. Boost.Voronoi serial tessellation library used. Timing profile for each step of the two-stage parallel algorithm.

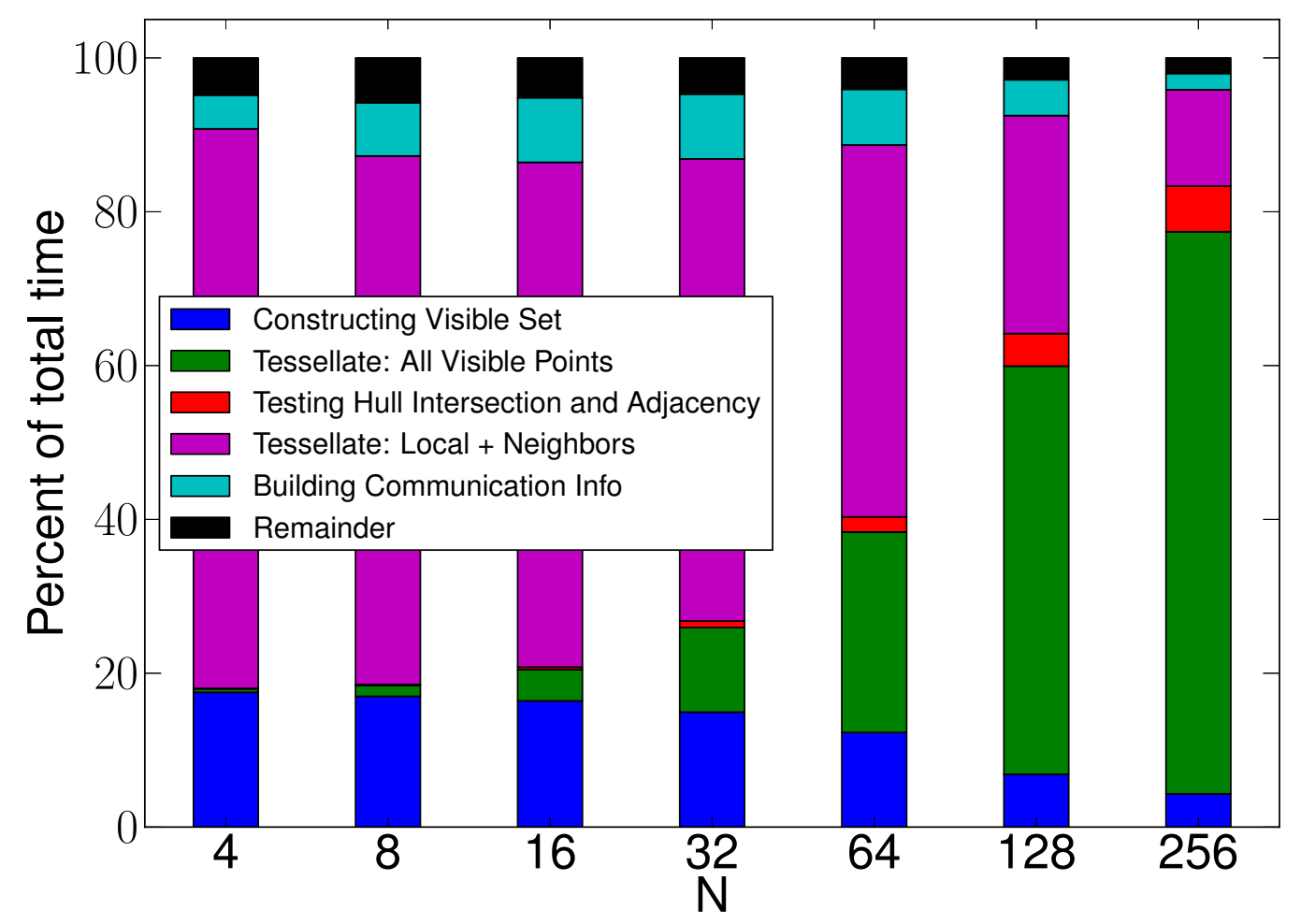

Figure 14: Parallel strong scaling of $10^{6}$ generators on a lattice distributed across $N$ processors with optimal decomposition. The five most expensive steps of the parallel algorithm broken down by percentage of total Voronoi construction time.

\section{Conclusions}

We present a novel procedure for constructing Voronoi diagrams from distributed input data. The algorithm is unique in that it computes a parallel communication layer for any 
arbitrary serial method for Delaunay or Voronoi grid generation. We have demonstrated the robustness of the algorithm on a variety of challenging two-dimensional geometries and input data. We have also provided initial scaling results which appear promising. Future work involves optimization of the algorithm as well as expansion of its testing into three dimensions.

The results presented in this paper were obtained using Polytope, an open-source library for the construction and storage of Voronoi diagrams as unstructured mesh data. Interested readers are encouraged to download the software and verify our results.

\section{Acknowledgments}

Our thanks to Misha Shashkov for many productive discussions on Voronoi mesh generation. This work was performed under the auspices of the U. S. Department of Energy by Lawrence Livermore National Laboratory under Contract DE-AC52-07NA27344.

\section{References}

[1] F. Aurenhammer, Voronoi Diagrams: A Survey of a Fundamental Geometric Data Structure, ACM Computing Surveys, 23(3), pp. 345-405, 1991.

[2] D. Blandford, G. Blelloch, C. Kadow, Engineering a Compact Parallel Delaunay Algorithm in 3D, Proceedings of the Twenty-Second Annual Symposium on Computational Geometry, p. 292, 2006.

[3] G. Blelloch, J. Hardwick, G. Miller, D. Talmor, Design and Implementation of a Practical Parallel Delaunay Algorithm, Algorithmica, 24, pp. 243-269, 1999.

[4] N. Chrisochoides, D. Nave, Parallel Delaunay Mesh Generation Kernel, Int. J. Numer. Meth. Engng., 58, pp. 161-176, 2003.

[5] R. Cole, M. Goodrich, C. Dunlaing, A Nearly Optimal Deterministic Parallel Voronoi Diagram Algorithm, Algorithmica, 16, pp. 569-617, 1996.

[6] Q. Du, V. Faber, M. Gunzburger, Centroidal Voronoi Tessellations: Applications and Algorithms, SIAM Review, 41(4), pp. 637-676, 1999.

[7] Q. Du, D. Wang, L. Zhu, On Mesh Geometry and Stiffness Matrix Conditioning for General Finite Element Spaces, SIAM J. on Numer. Anal., 47(2), pp. 1421-1444, 2009.

[8] S. Fortune, A Sweepline Algorithm for Voronoi Diagrams, Algorithmica, 2, pp. 153$174,1987$.

[9] R. Garimella, M. Shashkov, Polygonal Surface Mesh Optimization, Engineering with Computers, 20, pp. 265-272, 2004.

[10] B. Gehrels, B. Lalande, M. Loskot, A. Wulkiewicz, Boost.Geometry Library (Version 1.0), Available at http://www.boost.org (Accessed 7 June 2012). 
[11] D. Jacobsen, M. Gunzburger, T. Ringler, J. Burkardt, J. Peterson, Parallel Algorithms for Planar and Spherical Delaunay Construction with an Application to Centroidal Voronoi Tessellations, Geosci. Model Dev. Discuss., 6, pp. 1427-1466, 2013.

[12] R. Koradi, M. Billeter, P. Guntert, Point-Centered Domain Decomposition for Parallel Molecular Dynamics Simulation, Comp. Phys. Comm., 124, pp. 139-147, 2000.

[13] H. Ledoux and C. M. Gold, Modelling Three-Dimensional Geoscientific Fields with the Voronoi Diagram and its Dual, Int. J. Geographical. Inf. Sci., 22, pp. 547-574, 2008.

[14] H. Ledoux, Computing the 3D Voronoi Diagram Robustly: An Easy Explanation, Voronoi Diagrams in Science and Engineering 2007 Conference Proceedings, 2007.

[15] S. Lee, C.-I. Park, C.-M. Park, An Improved Parallel Algorithm for Delaunay Triangulation on Distributed Memory Parallel Computers, Parallel Processing Letters, $11,341,2001$.

[16] S. Lloyd, Least Square Quantization in PCM, IEEE Trans. Inform. Theory, 28, pp. 129-137, 1982.

[17] R. Loubere, P.-H. Maire, M. Shashkov, J. Breil, S. Galera, ReALE: A ReconnectionBased Arbitrary-Lagrangian-Eulerian Method, Journal of Computational Physics, 229, pp. 4724-4761, 2010.

[18] R. Merland and B. Levy and G. Caumon, Building PEBI Grids Conforming to 3D Geological Features Using Centroidal Voronoi Tessellations, IAMG 2011 Conference Proceedings, 2011.

[19] A. Okabe, B. Boots, K. Sugihara, S. N. Chiu, Spatial Tessellations: Concepts and Applications of Voronoi Diagrams, John Wiley and Sons, Chichester, West Sussex, England, 2000.

[20] J. M. Owen, Augmenting Meshless Methods Using the Voronoi Tessellation, SPHERIC Newsletter, 13, 2011.

[21] J. M. Owen, Applicatiions of the Voronoi Tessellation for Mesh-Free Methods, Multimat [Presentation], 2011.

[22] O. Pearce, T. Gamblin, B. de Supinski, M. Schulz, N. Amato, Quantifying the Effectiveness of Load Balance Algorithms. Int. Conf. on Supercomputing, pp. 185194, 2012.

[23] C. Rycroft, Voro++: A Three-Dimensional Voronoi Cell Library in C++, Chaos, 19, 041111, 2009.

[24] J. Shewchuk, Triangle: Engineering a 2D Quality Mesh Generator and Delaunay Triangulator, Applied Computational Geometry, 1148, pp. 203-222, 1996.

[25] J. Shewchuk, Adaptive Precision Floating-Point Arithmetic and Fast Robust Geometric Predicates, Discrete and Computational Geometry, 18(3), pp. 305363, 1997. 
[26] H. Si (2011), Tetgen (Version 1.4.3) . Available at http://tetgen.org (Accessed 28 January 2012).

[27] D. Sieger, P. Alliez, M. Botsch, Optimizing Voronoi Diagrams for Polygonal Finite Element Computations, Proceedings of the 19th International Meshing Roundtable, pp. 335-350, 2010.

[28] V. Springel, E Pur Si Muove: Galilean-Invariant Cosmological Hydrodynamical Simulations on a Moving Mesh, Mon. Not. of the R. Astron. Soc., in press, 2009.

[29] G. Taylor and A. Green, Mechanism of the Production of Small Eddies from Large Ones, Proc. Roy. Soc. A, 158, pp. 499-521, 1937.

[30] A. Sydorchuk (2010-2012), Boost.Polygon Voronoi Library (Version 1.0) . Available at http://www.boost.org (Accessed 25 March 2013).

[31] J. Wang, C. Cui, Y. Rui, L. Cheng, Y. Pu, W. Wu, Z. Yuan, A Parallel Algorithm for Constructing Voronoi Diagrams Based on Point-Set Adaptive Grouping, Concurrency Computat.: Pract. Exper., 2013.

[32] D.-M. Yan, B. Levy, F. Sun, W. Wang, Isotropic Remeshing with Fast and Exact Computation of Restricted Voronoi Diagram, Computer Graphics Forum, 28(5), pp. 1445-1454, 2009.

[33] D.-M. Yan, W. Wang, B. Levy, Y. Liu, Efficient Computation of 3D Clipped Voronoi Diagram, GMP 2010 Conference Proceedings, 2010.

[34] J. Johnson, J. M. Owen, D. Starinshak (2013), Polytope (Version 0.5.17). Available at https://bitbucket.org/jjphatt/polytope. 